19 Revue d'histoire du XIXe siècle

Société d'histoire de la révolution de 1848 et des

révolutions du XIXe siècle

$30 \mid 2005$

Pour une histoire culturelle de la guerre au XIXe siècle

\title{
Les sépultures de guerre en France à la fin du Premier Empire
}

Jacques Hantraye

\section{OpenEdition}

Journals

Édition électronique

URL : http://journals.openedition.org/rh19/1007

DOI : $10.4000 /$ rh 19.1007

ISSN : $1777-5329$

Éditeur

La Société de 1848

Édition imprimée

Date de publication : 1 juin 2005

ISSN : 1265-1354

Référence électronique

Jacques Hantraye, "Les sépultures de guerre en France à la fin du Premier Empire », Revue d'histoire du XIXe siècle [En ligne], 30 | 2005, mis en ligne le 28 mars 2008, consulté le 21 décembre 2020. URL http://journals.openedition.org/rh19/1007 ; DOI : https://doi.org/10.4000/rh19.1007

Ce document a été généré automatiquement le 21 décembre 2020.

Tous droits réservés 


\title{
Les sépultures de guerre en France à la fin du Premier Empire
}

\author{
Jacques Hantraye
}

1 En 1812, un jeune conscrit, Narcisse Faucheur, arrive à Napoléon-Vendée ${ }^{1}$ où il apprend qu'il doit remplacer un fourrier qui se meurt à l'hôpital. Lorsque ce militaire décède, un de ses amis demande à Faucheur de prononcer quelques mots sur la tombe du défunt, ce que le jeune homme accepte. Bien qu'ils n'aient pas pris part à la célébration, les officiers de l'unité sont gré au nouvel arrivant d'avoir honoré un camarade ${ }^{2}$.

Dans sa banalité, cette histoire met en évidence quelques-uns des enjeux liés à la mort des militaires à la fin du Premier Empire, après vingt années de conflits en Europe qui voient l'élaboration empirique d'une nouvelle culture de la mort à la guerre. Si elle rappelle que la majorité des militaires meurt encore à l'hôpital, et non sur le champ de bataille, cette anecdote suggère aussi que la mort est envisagée sans règles préétablies. Enfin, au-delà de la fonction d'intégration que remplissent les funérailles pour ce jeune soldat, elle souligne les liens qui existent entre civils et militaires dans ce domaine. On saisit aussi le paradoxe lié à l'inhumation des soldats au début du $\mathrm{XIX}^{\mathrm{e}}$ siècle: au-delà d'une apparente indifférence générale, se manifeste une réelle attention à l'égard de la sépulture, qui n'a pas toujours l'occasion de s'exprimer. Il s'agit ici d'analyser les liens qui existent entre les vivants et les morts et de déterminer quelle place occupent les défunts dans les attitudes et les pratiques des premiers, qu'ils soient civils ou militaires. Ces données prennent place dans un contexte qui n'est pas le même que celui que nous connaissons aujourd'hui, puisque les premiers cimetières militaires n'apparaissent en France qu'après $1870^{3}$. Ceci nous conduit à envisager successivement ce qui concerne le traitement du cadavre, puis les pratiques d'inhumation, avant d'évoquer les monuments funéraires et le souvenir des défunts. Les deux invasions étrangères qui ont entraîné la chute de Napoléon $\mathrm{I}^{\mathrm{er}}$ en 1814 et 1815 offrent un point de vue intéressant et assez inédit pour aborder ces questions.

3 Au soir de la bataille de Toulouse, le 11 avril 1814, le lieutenant anglais Woodberry écrit : la « quantité de cadavres autour de nous est énorme : il faut enterrer les morts ou quitter nos positions $»^{4}$. La vision des cadavres sans sépulture fait naître un effroi profond et 
suscite la répulsion. Il convient de s'en débarrasser. Des raisons d'hygiène et de salubrité ainsi que l'odeur du champ de bataille, que certains comparent à celle d'une boucherie, incitent à agir. Même en ville, comme à Troyes, l'odeur émanant des hôpitaux et des cimetières est insupportable ${ }^{5}$. Le lieutenant Gleig, du $85^{\mathrm{e}}$ régiment d'infanterie britannique, évoque un cas d'hallucination et de perte de connaissance chez une sentinelle obligée de passer la nuit auprès d'un mort, dans le Sud-Ouest, en janvier $1814{ }^{6}$. Il arrive même, à en croire cet officier, que des hommes préfèrent déserter plutôt que de subir cette cohabitation, au risque d'être fusillés pour abandon de poste. Or, les morts sont très nombreux. On relève ainsi 500 cadavres sous les murs de Grenoble en juillet $1815^{7}$. Beaucoup succombent également dans les hôpitaux militaires. À Huningue, dans le Haut-Rhin, c'est le cas de 566 hommes sur les 3480 que compte la garnison avant le début du blocus par les Alliés en 1814, la plupart étant morts du typhus ${ }^{8}$. Même des villes éloignées des combats sont concernées, car l'on y envoie des malades et des blessés. De 30 à 40 militaires décèdent chaque jour à l'hôtel-dieu de Paris entre février et avril $1814^{9}$

4 Et pourtant, en dépit de leur nombre, rares sont les défunts qui ne reçoivent pas de sépulture. Le voyageur britannique Stanley ne remarque qu'un seul cadavre lors de sa visite des champs de bataille picards et champenois en juillet $1814^{10}$. Toutefois, il arrive que certains corps soient enterrés avec retard. A Pontarlier, au début de 1814, on ne peut procéder à l'inhumation des nombreux militaires autrichiens morts de maladie, car la terre est gelée. Aussi les cercueils s'entassent-ils "par centaines » dans l'écurie d'une caserne, en attendant un moment plus favorable ${ }^{11}$. À Pantin, le 21 avril 1814, trois semaines après les combats qui accompagnent la chute de Paris, un habitant du lieu note que les cours et jardins «furent longtemps jonchés de morts » ${ }^{12}$. Les priorités du combat contraignent en effet à négliger les cadavres. Ceci impose de réfléchir aux attitudes manifestées à l'égard des morts. Comment les soldats envisagent-ils leur propre disparition et celle de leurs camarades ? Comment se met-on en règle devant la mort?

Le lieutenant britannique Gleig analyse finement son attitude et ses sentiments devant la mort, ainsi que ceux de ses compagnons d'armes, à la fin de 1813 et au début de 1814. Il observe que se protéger psychologiquement n'empêche pas d'éprouver des réactions $a$ posteriori. Un événement inhabituel impressionne plus que la mort au combat, à laquelle on est davantage préparé. Gleig, qui combat depuis plusieurs mois, fait ainsi des cauchemars à la suite d'un naufrage auquel il a assisté. Après la bataille, les survivants évacuent leurs angoisses en s'amusant. Ainsi, la vente des effets des camarades disparus, dévoilant ce qui fit leur intimité, suscite le rire, ce qui va de pair avec un sentiment de culpabilité à l'égard des morts. Ces attitudes ne sont contradictoires qu'en apparence ${ }^{13}$.

Les circonstances rendent les préparatifs du décès expéditifs. Ainsi, en février 1814, près d'Epernay, des soldats russes tués en représailles par des militaires et des civils français « demand[ent] seulement à recommander leur âme à Dieu avant de mourir " ${ }^{14}$. Il n'en est pas de même lorsque l'on voit venir la mort. À la suite du combat de La Rothière, en 1814, des officiers étrangers morts à l'hospice de Brienne ont le temps de remettre au curé des sommes au bénéfice de l'hospice " dans le but qu'on priât pour eux " ${ }^{15}$. Mais à l'hôpital, la mort est généralement solitaire et anonyme, et les cadavres ne sont guère respectés, de même qu'à la morgue ${ }^{16}$. Ceux qui se battent n'ont guère de possibilités de se préparer. Tout au plus l'officier Gleig consigne-t-il dans son carnet le nom de l'ami à qui il souhaite que ses affaires soient remises après sa mort ${ }^{17}$. Exceptionnel est le cas du commandant Otenin, du $136^{\text {e }}$ de ligne, commandant provisoire de la place de Compiègne lors de l'invasion de 1814. Lorsqu'il meurt, son décès est déclaré au bureau de l'état civil et le 
suppléant du juge de paix pose les scellés sur ses biens, confiés à un militaire de son régiment ${ }^{18}$

7 Cependant, les nécessités matérielles, qui poussent à agir vite, sont tempérées par des formes d'attention aux défunts. On peut relever en particulier une volonté d'identification des individus qui conduit à enregistrer les morts au combat, non pas en vue rapatrier les corps, mais pour des raisons administratives et par souci des familles. Lors des campagnes militaires, un officier est censé s'occuper de l'état civil de son unité, ce qui s'avère très difficile dans le chaos du champ de bataille ${ }^{19}$. Les administrateurs civils des hôpitaux à qui les militaires malades ou blessés sont confiés essaient de tenir à jour leurs registres, même s'ils sont débordés. A l'ambulance de Choisy-le-Roi, comme dans certains hôpitaux parisiens, on tient des registres séparés où l'on indique l'identité civile et militaire du défunt. À l'hôpital parisien de la Salpêtrière, par exemple, entre février et juin 1814, 1139 décès sont consignés, dont 89 concernant des anonymes ${ }^{20}$. Les maires sont également en charge de l'état civil des militaires. À Troyes, 3528 actes de décès sont enregistrés en 1814, contre 720 en 1815, mais les actes de décès de militaires concernent surtout ceux morts à l'hospice civil ${ }^{21}$. Dans la mesure du possible, ces documents sont adressés aux familles en France, voire à l'étranger. En Saône-et-Loire, l'administration préfectorale collecte à cet effet auprès des maires les pièces concernant les militaires étrangers ${ }^{22}$. En 1815, 4000 extraits d'actes mortuaires concernant des Espagnols sont expédiés à Madrid ${ }^{23}$. En juin 1816, le gouverneur de la province d'Anvers informe la population qu'il a reçu des attestations concernant des militaires morts au service de l'armée française sous la Révolution et l'Empire. Le grand nombre de défunts empêche toutefois de mener à bien cette procédure de façon systématique. Comprendre ces pratiques est essentiel pour analyser le traitement réservé au cadavre, car elles montrent que l'individu n'est pas négligé.

8 La sépulture est un fait culturel. On ne fait pas disparaître les cadavres n'importe comment. La question de la destruction des corps, accompagnée ou non de l'inhumation des restes, est complexe. Ainsi, la terre, la chaux et le feu peuvent avoir une fonction purificatrice qui n'est pas toujours perçue de façon négative ${ }^{24}$. Le 17 décembre 1813 , le préfet de Strasbourg donne l'ordre de brûler à l'écart des bâtiments de la ville les effets des militaires et la paille de leur literie ${ }^{25}$. La flamme sert à dissiper les odeurs, comme aux abords des creuttes d'Ailles, dans l'Aisne, où de nombreux corps ont été enterrés. Les chlorures de chaux et de soude, couramment répandus entre les lits des hôpitaux, servent à la désinfection ${ }^{26}$. Le feu est un moyen de détruire les corps peu mentionné dans les sources. Peut-être a-t-on souvent renoncé au bûcher uniquement faute de temps et de combustible. La crémation n'a pas forcément une connotation péjorative. Dans le département de l'Aube, on brûle des corps en 1814. À Waterloo aussi, après la bataille, 800 cadavres auraient été brûlés en un seul lieu. Toutefois, le bûcher, qui évoque à certains auteurs les traditions antiques, est inhabituel ${ }^{27}$. Le plus souvent on jette les corps à l'eau. Se débarrasser ainsi des défunts est une solution de commodité dans un pays où les cours d'eau abondent, motivée de plus par l'urgence. Après la bataille de Montereau, en 1814, on jette une partie des dépouilles dans la Seine ${ }^{28}$. Ce procédé correspond aussi à la volonté d'éliminer des traces compromettantes. En février 1814, après l'incendie d'un village, des militaires et des civils tuent par vengeance des soldats russes à proximité de la commune du Baizil afin d'éliminer les traces du crime. Ils font disparaître les corps dans un étang de la forêt de Vassy ${ }^{29}$. Pour des raisons d'hygiène, et peut-être de décence, les dépouilles trouvées dans les cours d'eau sont parfois inhumées après avoir été 
repêchées. Le 18 avril 1814, la Préfecture de police de Paris avertit les maires des communes riveraines que les corps trouvés dans la Seine doivent être enterrés sur place ${ }^{30}$ . En aval de Montereau, certains cadavres sont inhumés par des civils sur les rives du fleuve ${ }^{31}$

Mais l'eau et le feu n'ont pas qu'une fonction utilitaire. Lorsque l'ennemi dépasse les normes de violence admises, les civils et les militaires lui infligent un traitement particulier, qu'il soit mort ou même mourant. Celui qui n'est plus considéré comme un semblable est puni par le feu, mais surtout par l'eau. Le refus de sépulture constitue le reflet du combat : en un prolongement de l'affrontement, il est lié au franchissement d'un seuil de violence. Ces pratiques transgressives, entre lesquelles on peut distinguer des degrés, surviennent à des moments de paroxysme, notamment lors de l'invasion. À Melun, en février 1814, le cadavre d'un cavalier wurtembergeois est jeté par-dessus un pont avant d'être enterré ${ }^{32}$. On entend sans doute signifier à ses camarades qu'il est entré dans la ville de façon inacceptable, sans qu'il y ait eu négociation. Plus violent, après la victoire française de Bar-sur-Aube, des militaires étrangers crèvent les yeux de deux conscrits français avant de les jeter vivants à la Seine, à Clérey ${ }^{33}$. Les 7 et 8 mars 1814, après la bataille de Craonne, des militaires russes tentent d'enfumer des civils réfugiés dans des creuttes. En représailles, des hommes et des femmes de la région enterrent ou brûlent vifs des blessés ennemis. Certains de ces militaires brandissent des croix de paille pour indiquer qu'ils sont chrétiens. Ces pratiques suscitent l'indignation des habitants des environs, ce qui prouve qu'une telle attitude ne va pas de soi, même dans un contexte de grande violence ${ }^{34}$. Le 18 février 1814, un habitant de Montmirail met le feu à l'hôpital temporaire. Environ 500 militaires russes seraient morts asphyxiés, malgré les secours apportés par les habitants, qui auparavant avaient pris soin de l'ennemi. À Nogent-surSeine, le 17 mars 1814, ce sont des combattants russes qui, après avoir forcé les habitants à enterrer les morts de l'hospice, font brûler le bâtiment renfermant les survivants ${ }^{35}$. Le discours qui accompagne ces pratiques nous est parfois parvenu. On dit des morts jetés à l'eau qu'ils servent de «nourriture aux poissons » ou qu'ils vont annoncer aux Parisiens la défaite des envahisseurs, comme l'écrit le notaire H. G. Nicolet, historien de Melun sous la monarchie de Juillet ${ }^{36}$. À la fin de décembre 1813, dans le Sud-Ouest, Gleig remarque à propos des corps de militaires qu'il aperçoit dans un cours d'eau, qu'ils ressemblent à des paquets de linge ${ }^{37}$. On rapporte également qu'en février 1814, des pillards cosaques tués près de Nogent-sur-Seine sont retrouvés dans la Seine peu après, liés ensemble. Une perche, plantée au milieu d'eux comme un mât, porte un écriteau : «Laissez passer ces messieurs, ils vont directement à Paris ${ }^{38}$. L'anecdote, rapportée par un auteur fiable, constituerait, si elle est exacte, la seule mise en scène macabre connue à ce jour.

Ces récits montrent que le corps de l'ennemi est une arme destinée à terroriser et à dénier l'humanité de l'autre ${ }^{39}$. L'adversaire est réifié, animalisé, parfois diabolisé, y compris anéanti physiquement lorsque les comportements qu'on lui attribue sont réprouvés. En effet, les pratiques afflictives appliquées aux cadavres de ceux qui ont commis des crimes ou des délits prévus par la justice militaire sont généralement moins sévères. Ainsi, dans le Sud-Ouest, en 1813, deux déserteurs anglais sont fusillés sur le bord de la fosse destinée à les recevoir, avant que leurs compagnons d'armes ne défilent devant eux $^{40}$. À Reims, en mars 1814, le corps d'un civil, Gonze de Rougeville, fusillé pour trahison sur ordre d'une commission militaire française, est d'abord laissé sans sépulture à l'entrée du cimetière, avant d'être jeté à la fosse commune ${ }^{41}$. Si la tombe de ces 
individus est vouée à l'exécration et à l'abandon, leur corps n'est pas pour autant exhibé, mutilé ou anéanti au préalable.

11 Au-delà des exceptions que nous venons d'évoquer, les défunts sont majoritairement inhumés. Pour comprendre dans quelles conditions ils le sont, il faut prendre en compte le contexte de désorganisation dû à la guerre. Les autorités françaises sont parfois défaillantes. Ainsi, en juin 1814, l'adjoint au maire de Saint-Parre-les-Tartres, dans l'Aube, explique-t-il par les «malheurs » qu'a subi cette commune le fait que les corps de militaires et les carcasses de chevaux morts n'aient pas été enterrés. Dans ces conditions, c'est l'occupant qui prend le relais. Dans l'Aube, dès le 16 février 1814, le colonel Aunervadel, chargé de la police de la route par le prince de Schwarzenberg, ordonne au préfet de faire enterrer les chevaux morts qui encombrent les voies de communication ${ }^{42}$. On s'occupe également des cadavres de militaires. Que le sort des armes soit favorable aux troupes impériales, ou qu'un lieu soit momentanément épargné, et ce sont les autorités françaises qui font exécuter ces mesures. Le 3 mars 1814, le préfet de Seine-et-Marne donne ainsi l'ordre d'enterrer les corps trouvés dans son département ${ }^{43}$. Au cours du mois d'avril, le préfet de l'Aube presse plusieurs fois les sous-préfets d'enterrer les cadavres. Devant la réticence des ruraux, il recrute même du personnel pour accomplir cette tâche. Seize hommes, payés à raison de trois francs par jour, sont engagés en avril et mai $1814^{44}$.

12 Ces prescriptions sont dans l'ensemble appliquées. Ce sont souvent des particuliers qui se chargent d'enterrer les morts ou qui avancent les sommes nécessaires. À Arcis-sur-Aube, cinquante-deux notables se cotisent pour payer l'inhumation des militaires et l'enfouissement des chevaux du champ de bataille ${ }^{45}$. Ce sont parfois des soldats qui s'en chargent, ou encore les Frères des écoles chrétiennes, comme à Clacy, dans l'Aisne, en mars $1814^{46}$. L'opération suppose que l'on réquisitionne hommes, chevaux et véhicules, comme à Bosserville, près de Nancy ${ }^{47}$. Les hôpitaux embauchent parfois temporairement des fossoyeurs, comme à Meaux, où l'on en compte trois au printemps $1814{ }^{48}$. En ville, il arrive que l'on mette sur pied des commissions de salubrité composées du personnel médical civil du lieu, comme à Grenoble en 1815. Les avis que donnent ces médecins sont suivis ${ }^{49}$. On vérifie ultérieurement si les directives ont été appliquées. En mars et en avril 1814, des individus sont désignés par le préfet de l'Aube pour inspecter les routes et faire enterrer les corps. Le commissaire de police Claude Girardon, nommé en mai 1814, enquête dans un rayon d'une quinzaine de kilomètres autour de Troyes pendant 35 jours, inspectant au moins 22 communes ${ }^{50}$. Celles qui n'obéissent pas sont menacées de sanctions financières. La surveillance s'exerce en détail. A la Petite Villette, près de Paris, à la suite des combats de la fin mars 1814, plusieurs militaires morts sont enterrés dans la cour d'une maison. Le $1^{\text {er }}$ juillet suivant, le juge de paix fait recouvrir de terre les "débris » de ces "malheureux», sans doute par décence, mais aussi car leur présence "peut devenir un foyer de corruption et infecter l'air par des miasmes putrides et pestilentiels ${ }^{51}$. Il prescrit d'alerter les autorités locales.

13 La localisation de la sépulture est une donnée essentielle. Les morts inhumés rapidement, sur le lieu du combat ou ailleurs, sont souvent négligés. Ainsi, trois hussards autrichiens tués entre Toulon-sur-Arroux et Perrecy, en Saône-et-Loire, le 26 mars 1814, sont enterrés sur le bord de la route ${ }^{52}$. Sur le champ de bataille, c'est le sort de la plupart des dépouilles. À Waterloo, en 1816, une voyageuse britannique note que champs et fossés ne semblent former qu'une seule et même tombe ${ }^{53}$. Le lieutenant d'infanterie Lemet, tué à la bataille de Toulouse en 1814, est enterré dans un fossé et gît " oublié », déplore Carme- 
Duplan sous la Restauration. À Toulouse également, un officier britannique sollicite une trêve pour évacuer des cadavres entassés dans un secteur. Les Français concèdent une heure, pendant laquelle les adversaires conversent et boivent ensemble ${ }^{54}$

Au-delà de l'aspect pratique, inhumer sur le lieu de l'affrontement peut avoir un sens symbolique. Après une bataille, les visiteurs observent scrupuleusement la localisation des corps. Si les combattants sont tombés là où ils avaient reçu l'ordre de se tenir, c'est qu'ils se sont battus avec ténacité et courage ${ }^{55}$. Lors du blocus de Sélestat, en Alsace, le 6 mars 1814, une sortie entraîne la mort d'une cinquantaine d'hommes. Deux officiers du $40^{\mathrm{e}}$ de ligne tués ce jour-là sont enterrés dans une position avancée du dispositif fortifié. Sur ordre du commandant de place, on organise une cérémonie afin d'inciter les survivants à poursuivre la lutte ${ }^{56}$. De même, Carme-Duplan signale qu'une partie des militaires morts à la bataille de Toulouse en avril 1814 repose de part et d'autre d'un canal « comme pour faire entendre aux siècles à venir qu'ils furent ennemis " ${ }^{57}$. La tombe perpétue le combat, le corps du combattant enterré aux avant-postes étant censé rappeler la nécessité de ne pas céder du terrain et de venger les disparus.

Il n'existe pas de règles concernant l'inhumation proprement dite. Alors qu'au début du XIX ${ }^{e}$ siècle les autorités incitent à éloigner les morts du centre des localités, en 1814 les fosses sont situées hors de la ville, comme à Choisy, ou au contraire dans la cour d'un hôpital établi au cœur du bâti, ce qui est le cas à Meaux ${ }^{58}$. Dans les places fortes, l'Église veut que les cimetières soient clos, tandis que les militaires exigent que l'espace soit systématiquement dégagé pour faciliter la défense du lieu ${ }^{59}$. À Troyes, ce n'est que lorsque le cimetière communal est saturé que l'on a recours à des terrains vacants situés à la périphérie de la ville. Après la bataille de Montmirail, seuls les hommes tombés le plus loin des villages sont sommairement enterrés. On en dépose notamment dans un ravin ${ }^{60}$. À Bosserville, on vide et on aménage d'anciens étangs situés dans un bois dépendant d'une chartreuse abandonnée ${ }^{61}$. Il arrive que les fosses soient réservées aux militaires, comme à Meaux ou à Grenoble, ou bien qu'elles servent à la fois à la troupe et aux pauvres $\mathrm{du}$ lieu, comme à Troyes. Les fosses communes peuvent renfermer un grand nombre de cadavres. À Craonne, on aurait enterré 3000 hommes dans une même tombe, ce qui paraît beaucoup. Après la bataille de Toulouse, on parle de 700 corps enterrés ensemble ${ }^{62}$

Les pratiques liées à l'inhumation constituent un compromis entre l'attention à l'égard des défunts et la prise en compte de contraintes matérielles qui poussent à agir vite. Certes, le sort de la plupart est la sépulture d'urgence, en règle générale la fosse commune. Mais inhumation collective ne signifie pas forcément négligence à l'égard des cadavres. Au cours de sa tournée d'inspection, en juin 1814, le commissaire de police de Troyes établit une distinction entre les hommes, qui sont "inhumés », et les chevaux ou les bœufs qui sont "enfouis». Sur 891 bêtes enterrées, au moins 39 le sont de façon incomplète. En ce qui concerne les hommes, le rapport est de 8 pour 534, sans compter environ 800 corps jetés à la rivière ${ }^{63}$. Ce qui a trait aux humains n'est contradictoire qu'en apparence. En effet, si l'urgence après le combat amène à jeter les corps des militaires morts dans un cours d'eau, dès que l'occasion se présente on choisit de les enterrer avec un minimum de dignité, quitte à abandonner les bêtes à l'air libre. Quant à la sépulture collective, elle ne signifie pas nécessairement une forme de rejet. À Grenoble, les Austro-Sardes blessés devant la ville en juillet 1815 sont soignés par les habitants, avant même la capitulation française. Les morts n'en sont pas moins ensevelis dans une fosse commune. En effet, la sépulture collective est alors admise ${ }^{64}$. Elle est même parfois valorisée. Gleig ne présente-t-il pas la terre comme la «mère commune » ${ }^{65}$ ? La tombe 
collective est à l'image de l'hôpital, qui reçoit des hommes de toutes les origines, comme à Tournus en $1814^{66}$. À Bosserville sont inhumés dans le même secteur un prisonnier de guerre russe, un Italien au service de la France, des soldats nés en France et des employés de l'hôpital. Pougiat note que la fosse utilisée à Troyes en 1814 rassemble hommes et femmes, militaires et civils de toutes religions inhumés là sans la présence du clergé ${ }^{67}$. Au début des années 1830, dans un contexte d'anticléricalisme, l'auteur se saisit de cette observation pour inciter les prêtres catholiques à davantage de tolérance.

17 En temps normal, les civils honorent les combattants morts. Un simple soldat reçoit généralement une sépulture décente dans un cimetière, en présence d'un prêtre. Il en est ainsi à Brienne pour un conscrit de la Sarthe, le 22 décembre 1813, alors que des considérations sanitaires poussent à agir rapidement. C'est d'autant plus vrai pour des gradés. En janvier et en février 1814, à Brienne encore, des officiers sont accompagnés à leur dernière demeure par leurs pairs. Le général François Louis Forestier (1776-1814), notamment, est conduit à sa sépulture par son aide de camp. Les officiers reçoivent généralement un traitement particulier. Le général Eloi Taupin, mort de ses blessures le 11 avril 1814, est déposé dans l'église Saint-Etienne de Toulouse "pour être enterré le lendemain avec tous les honneurs de la guerre ${ }^{68}$.

Dans les moments troublés, les difficultés s'accumulent. Outre la désorganisation due aux combats, individus et localités ont peu de ressources, surtout à la fin de l'Empire, quand les charges se font plus lourdes. Parfois, pour des questions financières, les municipalités se montrent réticentes. C'est le cas de celle de Mâcon à l'égard d'un lieutenant du $50^{\mathrm{e}}$ d'infanterie mort en août 1814, alors qu'il était de passage en ville. Les autorités militaires et quelques officiers sont contraints de puiser dans la somme que le mort a sur lui ${ }^{69}$. La hâte et le manque d'argent n'empêchent pas la piété à l'égard des défunts. Après le combat d'Urrugne, dans le Sud-Ouest, en novembre 1813, les morts britanniques reçoivent une sépulture rapide, mais avec autant d'attention que le permettent les circonstances, selon ce que rapporte le lieutenant Gleig. Ils sont inhumés dans une même tombe, près de l'église, avec un "pieux respect ». Le lendemain, avant son départ, Gleig vient se recueillir sur la fosse avec deux ou trois camarades et y revient un mois après, à l'occasion d'un passage dans la région. Il souligne le «privilège » de ces hommes d'avoir reçu une sépulture ${ }^{70}$. Lors de l'inhumation, les corps peuvent être encore habillés, comme à Meaux ${ }^{71}$. À Choisy, au contraire, ils sont enterrés sans leurs effets militaires ${ }^{72}$. A Grenoble, pour des raisons sanitaires, on veille à disposer les cadavres entre deux lits de chaux de 40 centimètres d'épaisseur. Les derniers corps sont couverts de chaux, puis de 40 à 50 centimètres de terre ${ }^{73}$. À Choisy, en 1814, un militaire inhumé « de sa bourse » a sans doute droit à un cercueil. Pour ses compagnons plus démunis morts à l'ambulance, les frais de sépulture semblent être pris en charge par la municipalité ${ }^{74}$. Si l'urgence fait qu'à Bosserville on enterre vite, y compris des mourants, les ruraux requis ont parfois des attentions. Ainsi, ils prennent soin de réunir la famille d'une cantinière dans une même tombe ${ }^{75}$.

Reste à envisager plus précisément certaines grandes cérémonies funèbres, ainsi que le souvenir des défunts. Les célébrations accompagnant l'enterrement sont l'occasion d'exprimer un discours autour de la mort au combat, à mi-chemin des cérémonies funèbres de l'ère révolutionnaire et du rituel politique d'opposition de la Restauration ${ }^{76}$. Les funérailles revêtent dans certains cas un sens politique. Le comte Guillaume de SaintPriest, blessé devant Reims, meurt à Laon en mars 1814. D'après certaines sources, il est enterré solennellement dans une chapelle de la cathédrale, selon le rite orthodoxe, ce que 
les contemporains français perçoivent comme une marque d'extranéité. Significativement, le tombeau de cet émigré venu combattre son pays d'origine, élevé par son frère en août 1814 , est détruit sous Louis-Philippe ${ }^{77}$. Si, dans ce cas, les funérailles séparent les adversaires, il arrive au contraire qu'elles rapprochent occupants et occupés. De telles célébrations peuvent constituer un gage d'apaisement. À Soissons, une partie de la matinée du 15 février 1814, juste après la première reddition de la place, voit se dérouler les funérailles du général français Jean-Baptiste Rusca, mort en défendant la ville, à qui les troupes russes de Winzingerode rendent les honneurs militaires. La cérémonie se déroule suivant des règles définies sous l'Ancien Régime. Le cercueil, sur lequel sont posées les décorations, ainsi que les épaulettes et l'épée du défunt, est conduit à travers la ville depuis la maison mortuaire jusqu'au cimetière. L'assistance, composée de militaires et de civils, est très nombreuse. Des tirs d'artillerie et de mousqueterie accompagnent la mise en terre. Les habitants interprètent cet hommage comme un signe de bonne volonté à leur égard ${ }^{78}$.

Dans quelle mesure le souvenir se maintient-il après la cérémonie? Peu d'édifices funéraires datant du Premier Empire subsistent de nos jours, et les relevés effectués aux $\mathrm{XIX}^{\mathrm{e}}$ et $\mathrm{XX}^{\mathrm{e}}$ siècles sont peu nombreux. On se contentera donc de présenter quelques pistes à ce sujet. Notons tout d'abord que le fait de construire ou non un monument dépend beaucoup du lieu où est survenu le décès, un militaire mort en ville ayant plus de chances de se voir ainsi honoré. En 1814, parmi trois officiers supérieurs étrangers morts à Brienne, deux succombent sur le champ de bataille, tandis que le troisième meurt de ses blessures à l'hôpital. Or, seuls les restes de ce dernier sont inhumés au cimetière, dans un lieu signalé pendant plusieurs mois par une croix de bois. Les autres sont enterrés dans les champs, près du lieu où ils moururent. La fosse commune de Troyes est signalée pendant quelques temps par une croix ${ }^{79}$. Certaines églises abritent des stèles. À Waterloo, des épitaphes d'officiers anglais, de facture maladroite, sont installées par les régiments dans l'église du village ${ }^{80}$. La fonction des monuments est à la fois d'honorer les morts et d'exprimer une solidarité avec les vivants. Des civils prennent soin de maintenir la mémoire des militaires dont ils se sont occupés. À Montmirail, par exemple, une plaque évoque le souvenir de Charles Simon, lieutenant de la Landwehr de Silésie mort en février 1814. Cet homme, négociant dans le civil et peut-être d'origine française, meurt un mois après la bataille de Montmirail, chez un habitant de la localité ${ }^{81}$

21 La forme des monuments obéit aux canons du temps. À partir de la fin du XVIII ${ }^{\mathrm{e}}$ siècle, les sépultures militaires s'inspirent du style néo-classique ${ }^{82}$. Certaines constructions font penser aux cénotaphes à l'antique qui figurent parmi les fabriques des parcs de la bourgeoisie ou de l'aristocratie. C'est le cas de celui élevé au lieutenant-colonel Forbes, appartenant à un régiment d'infanterie britannique, mort le 10 avril 1814 sur le champ de bataille de Toulouse. On élève sur le lieu de sa mort un monument avec une inscription en anglais et en français célébrant son courage au combat. Le monument - une colonne surmontée d'une urne - du major Jacques Harrisson Baker, du $34^{\mathrm{e}}$ régiment d'infanterie anglais, blessé à la bataille de Toulouse, est édifié dans le jardin de M. Dargicourt, chez qui il est mort ${ }^{83}$. À Reims, les tombeaux d'officiers français, issus des couches supérieures de la société, sont édifiés en 1814 et en 1826 , date à laquelle des réinhumations ont lieu ${ }^{84}$.

La sépulture a non seulement une esthétique, mais un sens. Le tombeau, symbole de l'échange entre vivants et morts, intervient en paiement d'une dette contractée par la patrie. Il indique aussi un exemple à imiter. Comme l'écrit Carme-Duplan sous la Restauration, «[1]e simple soldat, en mourant, descend dans la tombe de l'oubli qui 
dévore jusqu'à sa mémoire. C'est la condition humaine de tout homme qui ne s'élève pas au-dessus de ses semblables. Mais celui qui s'illustre par ses vertus, ou qui donne au monde le spectacle honorable de grandes qualités, [...] échappant à la nuit des temps, reçoit en quelque sorte une seconde vie » ${ }^{85}$. À propos d'un lieutenant français mort à la bataille de Toulouse, il écrit que le monument édifié en hommage à son "dévouement " et à son " courage ", enseigne " qu'on doit toujours être prêt de mourir pour sa patrie " ${ }^{86}$. Il ne s'agit plus, comme sous la Révolution, d'honorer le soldat-citoyen mort pour la liberté ${ }^{87}$. On est désormais convaincu que la vertu au combat est surtout le fait des officiers, ce qui lie valeur militaire et appartenance aux élites sociales.

La tombe manifeste aussi la solidarité entre compagnons d'armes. Un jeune sous-officier irlandais apprécié de ses compagnons d'armes est tué au combat devant Bayonne au début de 1814. Après l'enterrement, ses camarades plantent un pin sur sa tombe et organisent une collecte en faveur de sa compagne. En 1815, un franc-tireur des environs de Pontarlier est tué lors d'une attaque. Ses camarades s'occupent de sa sépulture, puis de retour chez eux, vendent le butin pris à l'ennemi et partagent la somme obtenue avec la veuve de leur ami ${ }^{88}$. À Chaumont, en mars 1814 , l'inscription en russe sur le tombeau du lieutenant-colonel Blamow qualifie le défunt de "frère d'armes" ${ }^{89}$. À Reims, comme à Toulouse, les épitaphes évoquent l'échange qui unit le mort aux vivants: les qualités guerrières et le sacrifice sont contrebalancés par le repos dont jouit le disparu, ces mentions se concluant parfois par un appel à la prière.

Une fois les cérémonies terminées, la sépulture est parfois abandonnée, ce qui n'est pas propre aux seules tombes militaires. À Meaux, en 1814, les corps sont inhumés dans des fosses communes dans la cour de l'hôpital temporaire. L'édifice est affecté par la suite à l'armée. En 1820, à l'occasion de travaux d'aménagement, les restes sont éparpillés dans la cour ou portés dans la campagne sur l'ordre d'un capitaine du génie. Les ouvriers manient les ossements sans respect, tandis que les médecins civils envisagent seulement leur caractère nocif pour la santé. À Grenoble, en 1887, on retrouve fortuitement le lieu de sépulture des Alliés morts en 1815. Leurs restes sont aussi déplacés sans précautions. À Troyes, la tombe d'un canonnier étranger enterré en février 1814 au pied des remparts reste visible jusqu'à ce que des travaux de terrassement la fassent disparaître trois ans plus tard ${ }^{90}$. L'opposition entre fosse commune et tombe individuelle ne suffit donc pas à rendre compte de ce qui concerne la sépulture des militaires. Certaines tombes subsistent longtemps. Dans le cimetière de Chaumont, le monument du lieutenant-colonel russe Blamow, mort en 1814, est encore visible en 1868. Entre Biarritz et Arcangues, au début des années 1880 , on peut encore voir les pierres tombales de trois officiers britanniques ${ }^{91}$ . Il arrive que la présence d'une sépulture soit signalée par un toponyme, comme le champ des Cosaques - Kosakenfeld - à Sainte-Croix, près de Colmar, après le combat du 24 décembre 1813. Ce lieu fait naître la crainte : les riverains redoutent que ces individus morts de façon violente ne reviennent les hanter. À Montmirail, en 1814, des militaires tués lors de la bataille sont inhumés dans des carrières désaffectées, en un lieu appelé depuis le « bois-des-Cosaques ». Même abandonnée, la tombe suscite le malaise. Lors de sa visite à Waterloo en 1816, le Britannique Stanley, inquiet, se sent comme entouré par une armée de spectres ${ }^{92}$.

L'évolution de la mémoire inclut souvent une phase d'oubli. Ainsi, le contre-amiral Pierre Baste, mort à Brienne en janvier 1814, ne se voit pas élever de monument immédiatement. Sous la monarchie de Juillet, le gouvernement fait édifier sur sa tombe un «tombeau grec » en pierre comportant une épitaphe ${ }^{93}$. De même, le monument du 
général Rusca, au cimetière de Soissons, n'est érigé qu'en $1850{ }^{94}$. Le cas de Bosserville, en Lorraine, où plusieurs centaines d'hommes ont été inhumés en 1813 et 1814, illustre bien les fluctuations de la mémoire. Tout d'abord, un processus de désacralisation s'installe, qui se traduit par la plantation d'arbres à l'emplacement des fosses, ou encore par le fait que l'on manie les ossements sans ménagements. Pourtant, les fossoyeurs se souviennent précisément du jour et de l'endroit où ils ont procédé à des inhumations. En 1840, déjà, un professeur des environs s'intéresse au lieu. Vers 1905, l'intérêt renaît: des anciens parlent, le maire du village se surprend à feuilleter les vieux registres de décès, tandis qu'un comité se crée en vue d'élever un monument, à l'initiative du nancéien Émile Badel ${ }^{95}$. Après un temps de latence, les nouvelles générations relisent les événements en fonction d'enjeux nouveaux.

Sous le Second Empire, dans un climat plus apaisé, le clergé et les autorités civiles participent à la prise de conscience progressive. Le 11 février 1867, lors de l'inauguration du monument commémoratif de la bataille de Montmirail, l'évêque de Soissons évoque devant l'assistance les restes des disparus dispersés dans les alentours. Il célèbre ces individus morts pour la patrie, l'empereur et la France ${ }^{96}$. Sous la Restauration, ce sont parfois des femmes qui portent le deuil, mais dans l'espace public ce rôle leur est souvent refusé. Ainsi le monument que Claire Lenoir-Laroche, épouse d'un comte de l'Empire, a élevé à Sceaux, près de Paris, est-il fermé en $1820^{97}$. Les militaires jouent un rôle important. À Toulouse, Carme-Duplan propose ainsi d'élever un monument sur le lieu même des combats. En 1831, un prospectus annonce le lancement d'une souscription à Toulouse en vue d'édifier un monument « de deuil et de victoire ». L'avocat Romiguières, un des chefs locaux du parti libéral, fait partie du comité en compagnie de notables et de militaires. L'avocat Gasc évoque l'oubli des événements imposé par la Restauration et la volonté de célébrer « la mémoire du dévouement et du patriotisme » des guerriers ${ }^{98}$. Peu structurées à cause des vicissitudes politiques, essentiellement locales, les manifestations publiques du culte des morts sont souvent le fait de libéraux ou d'ultras. La mémoire est éclatée, diffuse. L'État, pour sa part, ne peut ou ne veut pas prendre en charge des sépultures très nombreuses et dont le souvenir est lié aux luttes qui ont divisé la France. En somme, la précipitation, le caractère inédit des événements, l'absence de valorisation des morts sous l'Empire laissent la place à des initiatives individuelles. Mais le désir d'honorer les morts, de façon collective, est bien présent. Il faudra attendre une volonté politique forte, et un nouveau conflit pour qu'une politique des sépultures militaires s'instaure après 1871 .

Narcisse Faucheur, l'ancien conscrit de 1812, se rend en 1850 sur les champs de bataille de Saxe où il avait combattu, et se recueille devant les restes de militaires tombés au cours de la campagne de $1813^{99}$. Un cycle s'achève, à l'échelle d'une vie, qui correspond au temps nécessaire pour que le deuil s'accomplisse.

Le sort réservé aux morts est inséparable des conditions créées par le combat et de la façon dont on traite malades et blessés. Les militaires sont assimilés aux pauvres, sans pour autant être rejetés, ce qu'indique le cas complexe de la sépulture collective. L'exemple de Reims nous montre comment, en un même lieu, on traite de façon différente les dépouilles des simples militaires et des officiers, des traîtres et des héros. On distingue avant tout ceux dont on considère qu'ils ont eu une belle mort. En raison d'une conception aristocratique du combat, on célèbre surtout la mort des officiers, qu'ils soient ou non issus des classes aisées. Ceux qui ont failli sont sanctionnés. Entre les deux, 
on doit se contenter dans le meilleur des cas de soins sommaires, mais la volonté de prendre en compte les cadavres des défunts est bien présente, ne serait-ce que par les progrès de l'enregistrement des morts dus à l'instauration de l'état civil. Cette étude illustre également la difficulté de faire émerger une commémoration civique des morts de la guerre après 1815, en raison du poids de l'Eglise et d'une culture du pouvoir monarchique qui prennent peu en considération l'individu mort au combat, notamment par rejet du pouvoir impérial. Mais la multiplicité des commémorations locales souligne l'intensité de la demande d'un culte des morts à la guerre dès le début du XIX siècle. Elle met en évidence l'attitude des survivants, faite d'un sentiment de redevabilité empreint d'affliction et de respect.

\section{NOTES}

1.. Aujourd'hui La Roche-sur-Yon.

2. Narcisse FAUCHEUR, Mon histoire, Lille, Danel, 1989 ( $1^{\text {re }}$ édition. 1886), pp. 43 et 98-101.

3. Luc CAPDEVILA et Danièle VOLDMAN, Nos morts : les sociétés occidentales face aux tués de la guerre, $X I X^{e}-X X^{e}$ siècles, Paris, Payot, 2002, p. 12.

4.. Journal du lieutenant Woodberry, Paris, Plon, 1896, p. 197.

5.. Georges Robert GLEIG, De Saint-Sébastien à Bayonne. Journal de camgagne d'un officier subalterne de l'armée de Wellington, 1813-1814, traduit de l'anglais par Charles Guiard, Bayonne, Imprimerie de A. Lamaignère, 1884, p. 164 et CARME-DUPLAN, Précis historique de la bataille livrée le 10 avril 1814, Toulouse, Bernichet, s. d., $3^{\mathrm{e}}$ partie, p. 147. Arch. mun. Troyes : 4 H 15, note sur l'infection des lieux publics, 1814.

6.. Georges Robert GLEIG, ouv. cité, pp. 90 et 207-208.

7.. Albin GRAS, Grenoble en 1814 et 1815, Grenoble, Maisonville, 1854, p. 60.

8. Arthur CHUQUET, L'Alsace en 1814, Paris, Plon, 1900, pp. 255-256.

9.. POUMIÈS DE LA SIBOUTIE, Souvenirs d'un médecin de Paris, Paris, Plon, 1910, p. 133.

10.. Edward STANLEY, Before and after Waterloo, Londres, Fisher Unwin, 1907, p. 168.

11.. PATEL, Souvenirs des deux invasions de 1814 et 1815 dans l'arrondissement de Pontarlier, Pontarlier, Simon, 1865, p. 15.

12.. Arch. dép. Seine-Saint-Denis, 4 U 5/303, 21.4.1814.

13. Georges Robert GLEIG, ouv. cité, pp. 198, 151 et 154.

14.. Service Historique de l'Armée de Terre (SHAT) : M R 1184, mémoire sur la reconnaissance de la route d'Epernay à Sommesous, 1826.

15. F. E. POUGIAT, Invasion des armées étrangères, dans le département de l'Aube, Troyes, Bouquot, 1833, p. 91.

16.. Bruno BERTHERAT, « La Morgue de Paris », Sociétés et représentations, n 6, juin 1998, pp. 273-293.

17.. Georges Robert GLEIG, ouv. cité, pp. 175-176.

18.. Arch. dép. Oise, 4 UP 12/12.

19.. Luc CAPDEVILA et Danièle VOLDMAN, ouv. cité, p. 68. 
20.. Arch. dép. Val-de-Marne, E dépôt Choisy-le-Roi, 4 H 2, registre mortuaire, 1814. Arch. Assistance Publique, Paris : 7 Q 31. POUMIÈS DE LA SIBOUTIE, ouv. cité, p. 134. Une ambulance est un hôpital temporaire.

21.. Arch. mun. Troyes : commission administrative de l'hospice au maire, 11 juin 1814 ; registres des décès de 1814 et $2 \mathrm{M} 60$, statistique des décès.

22.. Arch. dép. Saône-et-Loire : $3 \mathrm{R}$ (actes de décès), maire de Mâcon au préfet, 7 juillet 1814.

23.. Archives de l'Etat, Anvers : Arch. prov., J, 161 (A), affiche, $1^{\text {er }}$ juin 1816. Jean-René AYMES, La déportation sous le Premier Empire. Les Espagnols en France (1808-1814), Paris, Publications de la Sorbonne, 1983, 568 p., p. 200.

24.. On brûle ou on enterre les drapeaux afin que l'ennemi ne s'en empare pas (Edward STANLEY, ouv. cité, p. 115).

25.. Paul L'HUILLIER, Le typhus de 1813-1814 à Strasbourg, Strasbourg, Éditions universitaires de Strasbourg, 1925, pp. 97-99.

26.. Édouard FLEURY, Le département de l'Aisne en 1814, Laon, Fleury, 1858, pp. 406-407. Paul L'HUILLIER, ouv. cité. p. 100.

27. F. E. POUGIAT, ouv. cité p. 52. Edward STANLEY, ouv. cité, p. 267. Voir aussi

L. CHEVALIER, Histoire de Bar-sur-Aube, Bar-sur-Aube, chez l'auteur, 1851, p. 266.

28. Paul QUESVERS, La bataille de Montereau, Montereau, Zanote, 1900, p. 64. Voir aussi

F. E. POUGIAT, ouv. cité, p. 141 et Édouard FLEURY, ouv. cité, p. 407.

29.. SHAT, 1 M 1184, mémoire sur la reconnaissance de la route d'Epernay à Sommesous, 1826.

30.. Arch. dép. Val-de-Marne, E dépôt Saint-Maurice, 1 i 6, chef de la $3^{\mathrm{e}}$ division de la Préfecture de police, 18 avril 1814.

31.. Gabriel LEROY, Histoire de Melun, Office d'édition du livre d'histoire, 1994 (1 ${ }^{\text {re }}$ édition 1887), p. 462.

32.. Idem, p. 460.

33. F. E. POUGIAT, ouv. cité, p. 171.

34.. Édouard FLEURY, ouv. cité, pp. 246-249.

35. Edgar GROSJEAN, Autour de la bataille de Montmirail, Châlons-sur-Marne, Martin frères, 1905, p. 31. Madeleine TARTARY, Épisode de la campagne de France : Nogent-sur-Seine en 1814, Paris, Éditions des Presses modernes, 1939, p. 162.

36.. Georges Robert GLEIG, ouv. cité, p. 174, et Journal du lieutenant Woodberry, ouv. cité, p. 245. H. G. NICOLET, Histoire de Melun, Péronnas, La Tour Gile, 1999 ( $1^{\text {re }}$ édition 1843), pp. 333-334.

37.. Georg Robert GLEIG, ouv. cité, p. 174.

38. J.-M. LEMAITRE, Combat de Nogent-sur-Seine, Nogent, Librairie des Petites-Affiches, 1840, p. 38.

39.. Voir Luc CAPDEVILA et Danièle VOLDMAN, ouv. cité, pp. 132 et 137.

40.. Georges Robert GLEIG, ouv. cité, pp. 90-93.

41. A. DRY, Reims en 1814 pendant l'invasion, Paris, Éditions Plon, 1902, pp. 166-172.

42.. Arch. dép. Aube, 14 R 67, adjoint de Saint-Parre-les-Tartres, $1^{\text {er }}$ juin 1814 et Aunervadel au préfet de l'Aube, 16.2.1814. Arch. Commun. Troyes : 4 H 29, 20 février 1814.

43.. Madeleine TARTARY, ouv. cité, p. 171.

44.. Édouard FLEURY, ouv. cité, p. 406. Arch. dép. Aube, 14 R 67, arrêté préfectoral, $1^{\text {er }}$ avril 1814 ; état des journées des ouvriers, 1814.

45.. Idem : état d'une somme versée, 25 mai 1814 et lettre du préfet, 8 juin 1814.

46.. Édouard FLEURY, ouv. cité, p. 304. 
47.. Émile BADEL, Le monument de Bosserville, 1911, 116 p., p. 6.

48. A M. Meaux, $4 \mathrm{H}$ 16, note sur les employés de l'ambulance, 17 avril 1814.

49. Albin GRAS, ouv. cité, p. 60.

50.. C. ARPIN, Les horreurs de la campagne de 1814 dans le département de l'Aube, Troyes, Grande imprimerie de Troyes, 1910, pp. 39-40 et 46. Voir aussi Arch. dép. Aube, 14 R 67, arrêtés des 17 mars et $1^{\mathrm{er}}$ avril 1814 et procès-verbaux, $1^{\mathrm{er}}$ juin au 23 juin 1814 .

51.. Arch. dép. Seine-Saint-Denis, 4 U 5/303, $1^{\mathrm{er}}$ juillet 1814.

52.. Arch. dép. Saône-et-Loire, $3 \mathrm{R}$ (actes de décès), maire de Toulon-sur-Arroux au souspréfet de Charolles, 7.7.1814.

53. Edward STANLEY, ouv. cité, p. 268.

54.. CARME-DUPLAN, ouv. cité, $3^{\mathrm{e}}$ partie, pp. 123 et 131-132.

55. A. CARRO, Histoire de Meaux et du pays meldois, Meaux, Le Blondel, 1865, pp. 471-472 et Edward STANLEY, ouv. cité, p. 116.

56. Arthur CHUQUET, ouv. cité, p. 192.

57.. CARME-DUPLAN, ouv. cité, p. 135.

58. Arch. mun. Meaux : 5 I 7, procès-verbal, 18 mars 1820.

59.. Madeleine LASSÈRE, Villes et cimetières en France de l'Ancien Régime à nos jours, Paris,

L'Harmattan, 1997, 411 p., p. 122.

60.. Edgar GROSJEAN, ouv. cité, p. 57.

61.. Émile BADEL, ouv. cité, p. 7.

62.. Edward STANLEY, ouv. cité, p. 164. CARME-DUPLAN, ouv. cité, $3^{\mathrm{e}}$ partie, p. 139.

63.. Arch. dép. Aube, 14 R 67, procès-verbaux, 1.6 au 23.6.1814. Cette comptabilité reste imprécise.

64.. C'est à partir de la guerre de 1870-1871 qu'elle fait l'objet d'un rejet (Danielle TARTAKOWSKY, Nous irons chanter sur vos tombes. Le Père-Lachaise, XIX ${ }^{e}$-XX $X^{e}$ siècle, Paris, Éditions Aubier, 1999, pp. 50 et 52). Albin GRAS, ouv. cité, pp. 60-61.

65.. Georges Robert GLEIG, ouv. cité, p. 171.

66.. Arch. dép. Saône-et-Loire, 5 E 543/17.

67. Émile BADEL, ouv. cité, p. 94, 99, 101 et 108. F. E. POUGIAT, ouv. cité, pp. 364-365.

68. Arch. dép. Aube : 112 J 38. CARME-DUPLAN, ouv. cité, $3^{\mathrm{e}}$ partie, p. 108.

69.. Arch. dép. Saône-et-Loire, 4 U 1885, 19 août 1814.

70.. Georges Robert GLEIG, ouv. cité, pp. 111 et 170.

71.. Arch. Commun. Meaux, 5 I 7, rapport, 9 novembre 1820.

72.. Arch. dép. Val-de-Marne : E dépôt Choisy-le-Roi, 4 H 1, lettre au maire de Choisy, 8 juillet 1814.

73.. CHARVET, Mémoire sur le lieu de sépulture des austro-sardes tués devant Grenoble en juillet 1815, Grenoble, Allier, 1887, pp. 5 et 6.

74.. Les frais sont compris entre un et deux francs par défunt (Arch. dép. Val-de-Marne, E dépôt Choisy : $4 \mathrm{H} 1$ et $4 \mathrm{H}$ 2, état des fournitures, état de frais de sépultures et registre des morts, sd, 1814).

75.. Émile BADEL, ouv. cité, p. 16.

76.. Emmanuel FUREIX, « Un rituel d'opposition sous la Restauration : les funérailles libérales à Paris (1820-1830) », Genèses, n² 46, mars 2002, p. 77.

77. Cet ancien de l'armée de Condé devint officier supérieur dans l'armée du tsar (Michaud, Biographie universelle, tome XXXVII, Paris, Desplaces, sd, 695 p., p. 413). J. DEVISME, Histoire de la ville de Laon, Paris, Office d'édition du livre d'histoire, tome 2, 1822, 1996, p. 225, Édouard FLEURY, ouv. cité, p. 332 et A. DRY, ouv. cité, p. 215. 
78.. Édouard FLEURY, ouv. cité, pp. 117-118. André CORVISIER, Les hommes, la guerre et la mort, Paris, Éditions Economica, 1985, p. 385. M. LEROUX, Histoire de la ville de Soissons, tome 2, Soissons, Fossé Darcosse, 1839, p. 410.

79. F. E. POUGIAT, ouv. cité, pp. 73 et 364-365.

80.. Edward STANLEY, ouv. cité, p. 261.

81.. Edgar GROSJEAN, ouv. cité, pp. 44-45.

82. André CORVISIER, ouv. cité, p. 387.

83.. CARME-DUPLAN, ouv. cité, pp. 37, 43-46 et 54-55.

84.. A. DRY, ouv. cité, pp. 224 et 364-365.

85.. CARME-DUPLAN, ouv. cité, $3^{\mathrm{e}}$ partie, pp. 124.

86. Idem, pp. 101-102.

87.. André CORVISIER, ouv. cité, pp. 387-388.

88.. Georges Robert GLEIG, ouv. cité, p. 256. PATEL, ouv. cité, pp. 38-39.

89.. F.-F. STEENACKERS, L'invasion de 1814 dans la Haute-Marne, Paris, Didier, 1868, pp. 204-207.

90.. Arch. mun. Meaux, 5 I 7, rapport, 9.3.1820. CHARVET, ouv. cité, p. 4. F. E. POUGIAT, ouv. cité, pp. 265-266.

91.. F.-F. STEENACKERS, ouv. cité, Paris, Didier, 1868, pp. 204-207, Georges Robert GLEIG, ouv. cité, pp. 295-296.

92. Arthur CHUQUET., ouv. cité, p. 47. Edgar GROSJEAN., ouv. cité, p. 57. Edward STANLEY, ouv. cité, pp. 264-265.

93. F. E. POUGIAT, ouv. cité, p. 71. BOURGEOIS, Histoire des comtes de Brienne, Troyes, Anner-André, 1848, p. 240 et Étienne TAILLEMITE, Dictionnaire des marins français, Paris, Éditions Tallandier, 2002, pp. 31-32.

94.. Édouard FLEURY, ouv. cité, pp. 117-118.

95.. Émile BADEL, ouv. cité, pp. 13, 14, 17, 22 et 44.

96.. Inauguration du monument commémoratif de la bataille de Montmirail, Châlons-sur-Marne, Martin, 1867, p. 11.

97.. Jacques HANTRAYE, La société française et la guerre, thèse sous la dir. de M. Alain CORBIN, Université Paris I, 2001, tome III, pp. 905-910.

98.. CARME-DUPLAN, ouv. cité, $1^{\text {re }}$ partie, p. 28. Souscription pour le monument du 10 avril 1814, Toulouse, 1831, np, et J. L. GAZZANIGA (dir.), Histoire des avocats et du barreau du Toulouse du XVIII siècle à nos jours, Toulouse, Privat, 1992, 328 p., p. 74. Discours prononcé par M. Gasc, Toulouse, de Caunes, 8 p., sd, pp. 2 et 4.

99.. Narcisse FAUCHEUR, ouv. cité, pp. 170-174.

\section{RÉSUMÉS}

La plupart des militaires morts à la fin du Premier Empire sont enterrés dans des sépultures collectives à cause du manque de moyens et de la désorganisation des instances administratives. Il n'en demeure pas moins que les autorités locales cherchent à les identifier, à des fins administratives, et que tous essaient généralement de procurer aux corps un mode de sépulture décent, quoique sommaire. Dès que la possibilité se présente, les militaires sont enterrés avec un 
certain cérémonial.Les officiers bénéficient d'un sort particulier. Le fait qu'ils appartiennent généralement aux classes aisées et le courage de certains au combat leur valent souvent des funérailles qui constituent un témoignage de reconnaissance à leur égard. L'enterrement d'officiers de haut rang revêt parfois un sens politique.La mémoire des défunts se construit difficilement, le plus souvent en marge des instances officielles, religieuses ou politiques, peu soucieuses après 1815 d'honorer le régime napoléonien. Pourtant, le besoin de commémorer les morts se fait jour, suscitant des initiatives locales, parfois à des fins politiques.

Burying the dead before the military graveyards : soldiers' funerals at the end of the Napoleonic era

Most of the soldiers and officers who died at the end of the Napoleonic era were buried in common graves. It does not mean that they were neglected. Soldiers and civilians tried to identify them for administrative purposes prior to burying operations. They took much care of the corpses, organizing decent funerals as frequently as they could. Though officers were the most honoured, foot soldiers were not left aside. Some of those celebrations were organized for political reasons.During the first half of the 19th century, the memory of fallen soldiers and officers had to face the reaction of State and Church after Waterloo and was taken on merely by veterans and liberals. Speeches, recollections of memories and monuments building show that the memory of dead soldiers was a very important matter, 50 years before the first military graveyard appeared.

\section{AUTEUR}

\section{JACQUES HANTRAYE}

Docteur de l'Université Paris I-Panthéon-Sorbonne (Centre de recherches en histoire du XIX ${ }^{\mathrm{e}}$ siècle) 